\title{
Cor Triatriatum Dexter: A Case Report in a 70-Year-Old Male
}

\author{
George Hanna ${ }^{\mathrm{a}}$, Javad Savoja, Syed Iftikhar ${ }^{\mathrm{a}}$, Patrick $\mathrm{Hu}^{\mathrm{b}, \mathrm{c}, \mathrm{d}}$
}

\begin{abstract}
Cor triatriatum dexter (CTD) is a rare congenital heart disease resulting from persistence of the right valve of the sinus venosus. The persistent valve forms a membrane that divides the right atrium into a proximal and a distal chamber. This disorder exhibits varying clinical manifestations depending on the degree of partitioning or septation of the right atrium. In asymptomatic patients, the disease may be discovered during surgical procedures, diagnostic testing such as echocardiography, or hemodynamic monitoring. Severe septation abnormalities may cause right-sided heart failure and elevated central venous pressures due to obstruction of the tricuspid valve, right ventricular outflow tract, or inferior vena cava. Here we report a case of CTD in a patient presenting with symptoms of dyspnea and fatigue, followed by a short discussion of the embryology and clinical implications of this congenital disease, as well as current advances in management.
\end{abstract}

Keywords: Cor triatriatum dexter; Right-sided heart failure; Congenital heart disease

\section{Introduction}

Cor triatriatum dexter (CTD) is an extremely rare congenital heart disease that results from the continued presence of the right valve of the sinus venosus. This valve forms a membrane that divides the right atrium into a proximal and a distal chamber [1]. This disorder has varying clinical manifestations, from asymptomatic disease to severe right-sided heart failure and elevated central venous pressures.

Manuscript submitted May 27, 2020, accepted June 6, 2020

Published online July 21, 2020

aDepartment of Internal Medicine, UC Riverside School of Medicine/Riverside Community Hospital, Riverside, CA 92501, USA

bDepartment of Cardiology, Riverside Medical Clinic, Riverside, CA 92501, USA

'Internal Medicine/Cardiology, Riverside Community Hospital, University of California, Riverside, CA 92501, USA

${ }^{\mathrm{d} C}$ Corresponding Author: Patrick Hu, Internal Medicine/Cardiology, Riverside Community Hospital, University of California, Riverside, CA 92501, USA. Email:dr.hu.md@gmail.com

doi: https://doi.org/10.14740/jmc3506

\section{Case Report}

A 70-year-old Asian male presented to the hospital with worsening dyspnea on exertion and severe fatigue. He also reported an "issue with his heart" and a murmur which had been present since childhood. The patient noted absence of chest pain, orthopnea, or palpitations. He had a past medical history consistent with essential hypertension and cataracts but denied any history of diabetes, dyslipidemia or smoking. Family history of cardiovascular-related disease was non-existent.

On examination, his vitals were stable. The patient weighed $45 \mathrm{~kg}$ with a body mass index (BMI) of $17.2 \mathrm{~kg} / \mathrm{m}^{2}$. Cardiovascular exam was significant for a $4 / 6$ systolic murmur with a palpable thrill best heard at the left lower sternal border and a right ventricular heave.

Transthoracic echocardiography was significant for a left ventricular ejection fraction (LVEF) of $65 \%$, as well as moderate to severe mitral and tricuspid regurgitation (Figs. 1, 2). Transesophageal echocardiography revealed a normal left ventricular size and systolic function with an LVEF of $55-65 \%$. It also showed evidence of a septum within the right atrium diagnosed as CTD. The mitral valve had moderate to severe regurgitation and the tricuspid valve had severe regurgitation. There was an intra-atrial septal aneurysm with a small patent foramen ovale and a right-to-left shunt (Figs. 3, 4).

Left heart catheterization was significant for a vertically rotated heart. He had several redundant large caliber vessels in the left circumflex and right coronary artery, in addition to a large caliber wrap-around left anterior descending coronary artery with no stenosis noted in these arteries, just luminal irregularities.

The patient was discharged to be followed up in the cardiology clinic for continued evaluation of his valvular disease and cor triatriatum. Together, they appeared to be the main contributors to his fatigue and shortness of breath. In contrast, his coronary arteries were large in caliber, with no disease that could be attributing to his symptoms. The patient would require monitoring for his mitral and tricuspid regurgitation with the possibility of surgical/percutaneous valve repair if deemed necessary in the future. He would also require monitoring for right ventricular failure secondary to CTD with the possibility of surgical resection/percutaneous disruption of the membrane if needed.

\section{Discussion}

CTD results from partitioning of the right atrium to form a tria- 


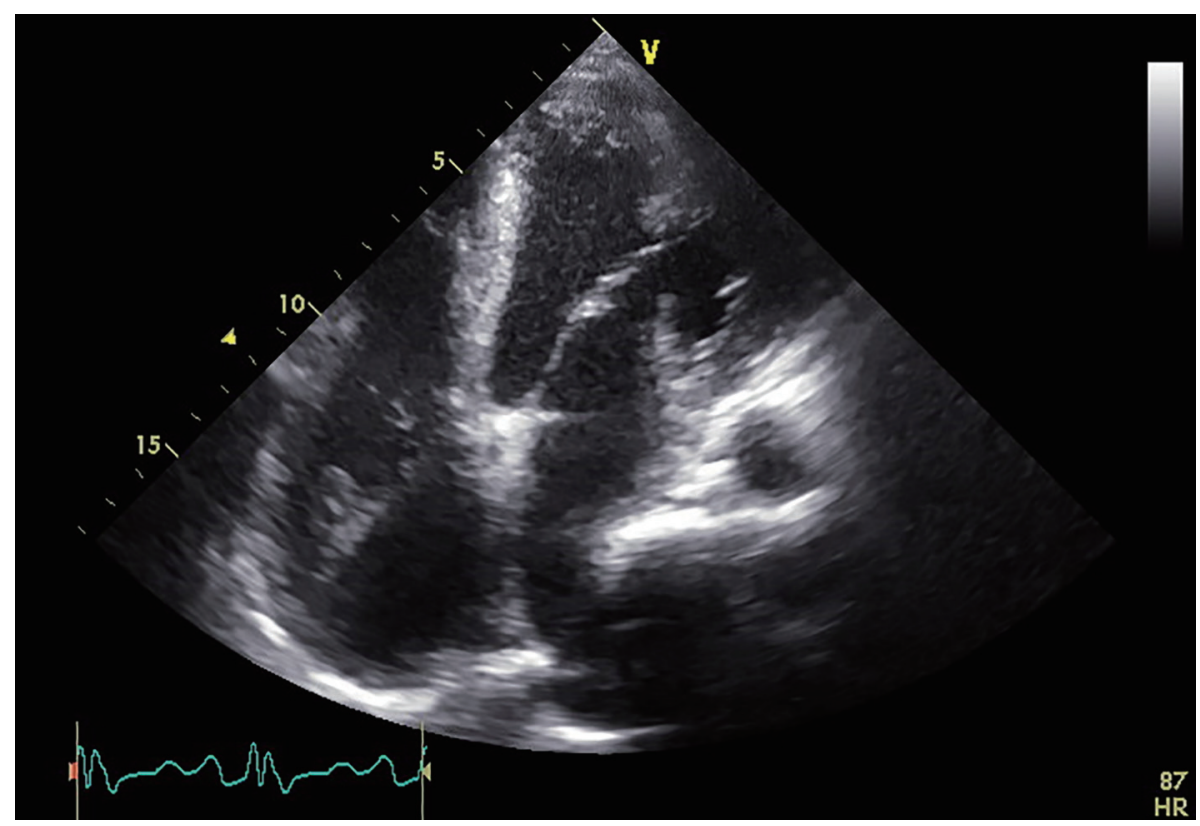

Figure 1. Transthoracic echocardiography in an apical four-chamber view revealing a left ventricular ejection fraction of $65 \%$ and a possible septation abnormality within the right atrium.

trial heart. Unlike cor triatriatum sinister (left atrial septation), which is more common, CTD is an extremely rare congenital anomaly. It has an incidence of approximately $0.1 \%$ of congenital heart malformations [2].

During embryogenesis, the right horn of the sinus venosus gradually incorporates into the right atrium to form the smooth posterior portion of the right atrium, whereas the original embryologic right atrium forms the trabeculated anterior portion. The right valve of the right horn of the sinus venosus divides the right atrium into two. This right valve forms a sheet that serves to direct the oxygenated venous return from the inferior vena cava across the foramen ovale to the left side of the heart during the fetal life [1]. Normally, the valve regresses at approximately 12 weeks gestation and leaves behind the crista terminalis proximally, and the eustachian valve of the inferior vena cava and the thebesian valve of the coronary sinus distally. Complete persistence of the right sinus valve results in a separation between the smooth and trabeculated portions of the right atrium, resulting in

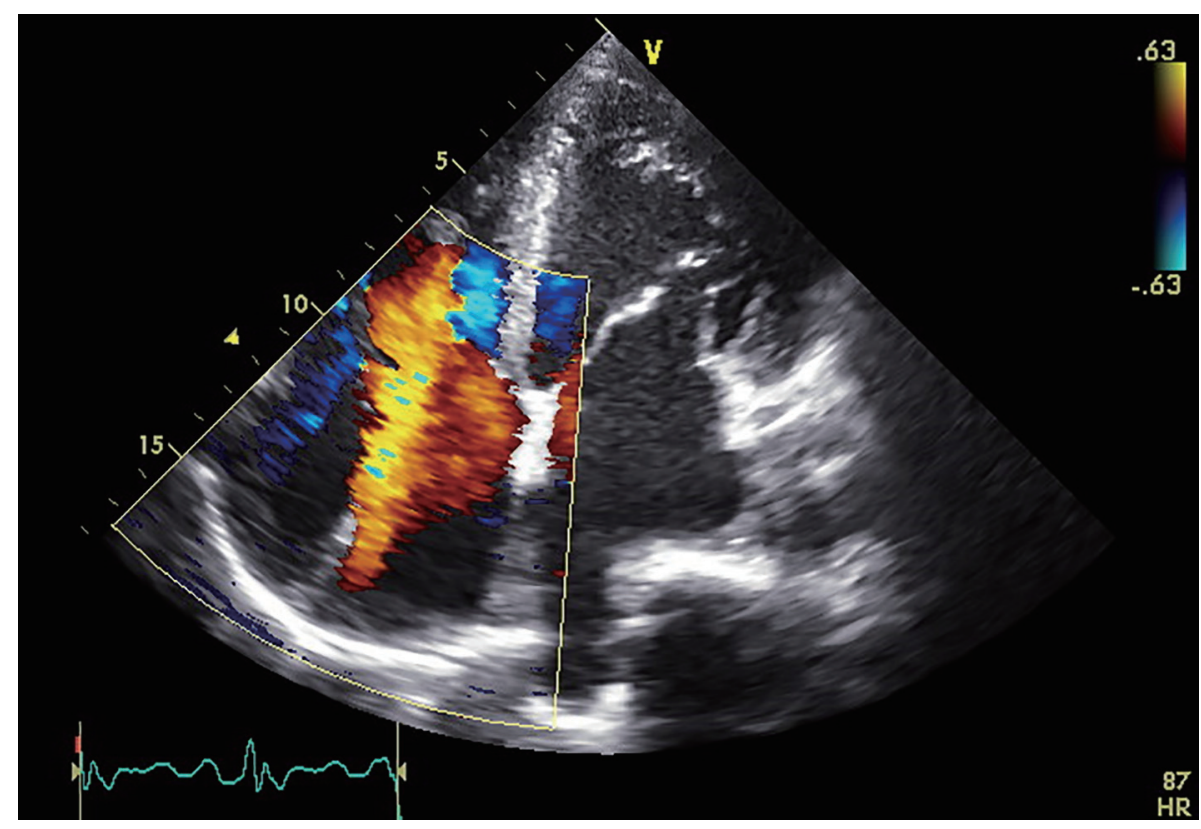

Figure 2. Transthoracic echocardiography in an apical four-chamber view with color Doppler revealing moderate to severe tricuspid regurgitation. 


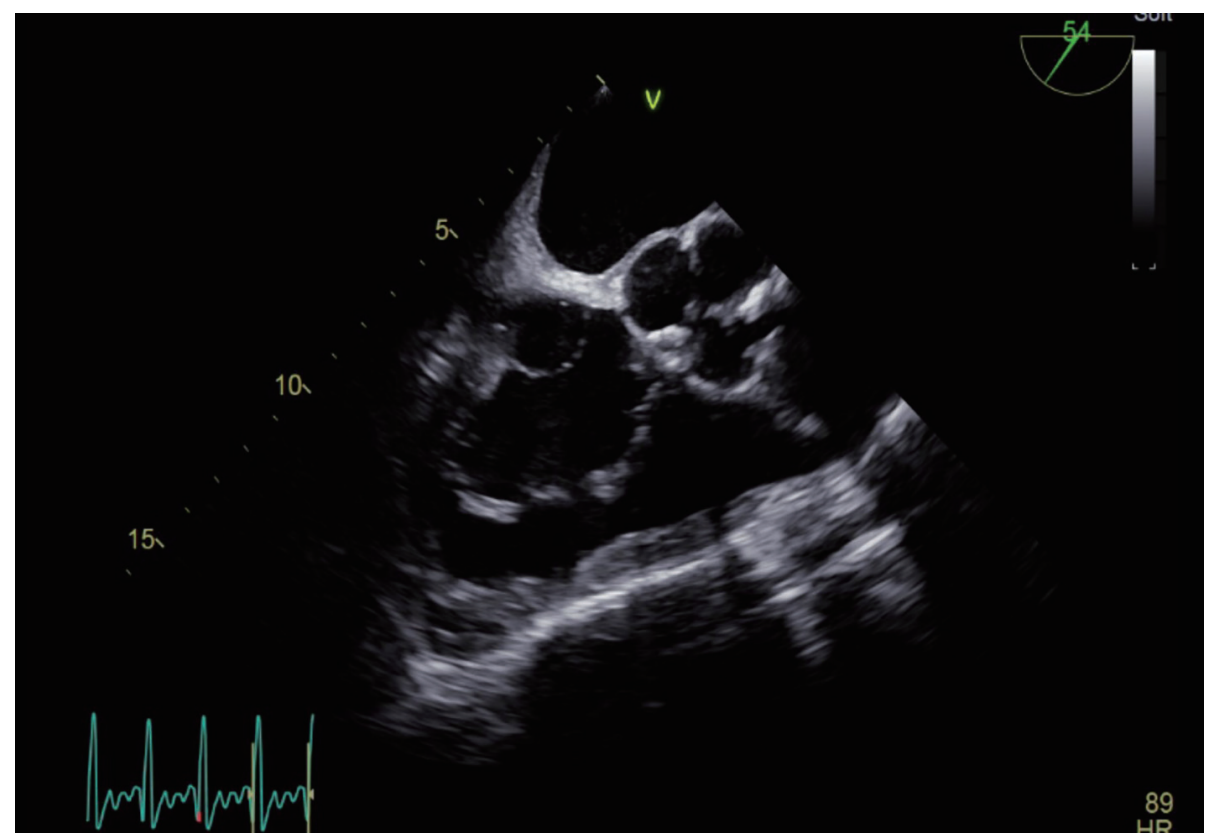

Figure 3. Transesophageal echocardiography at the level of the aortic valve with the scope rotated to $54^{\circ}$ showing evidence of a septum within the right atrium diagnosed as cor triatriatum dexter.

CTD. The proximal portion receives the venous blood from both vena cavae and the distal portion is in contact with the tricuspid valve and the right atrial appendage [3].

CTD can present in a myriad of forms depending on the degree of partitioning or septation/fenestration of the right atrium. When the septation is mild, the condition is often asymptomatic, and is found incidentally during surgery to correct other cardiac abnormalities or during echocardiography. More severe septation can cause right-sided heart failure and elevated central venous pressures due to obstruction of the tricuspid valve, the right ventricular outflow tract, or the inferior vena cava. CTD can occur as an isolated cardiac anomaly or

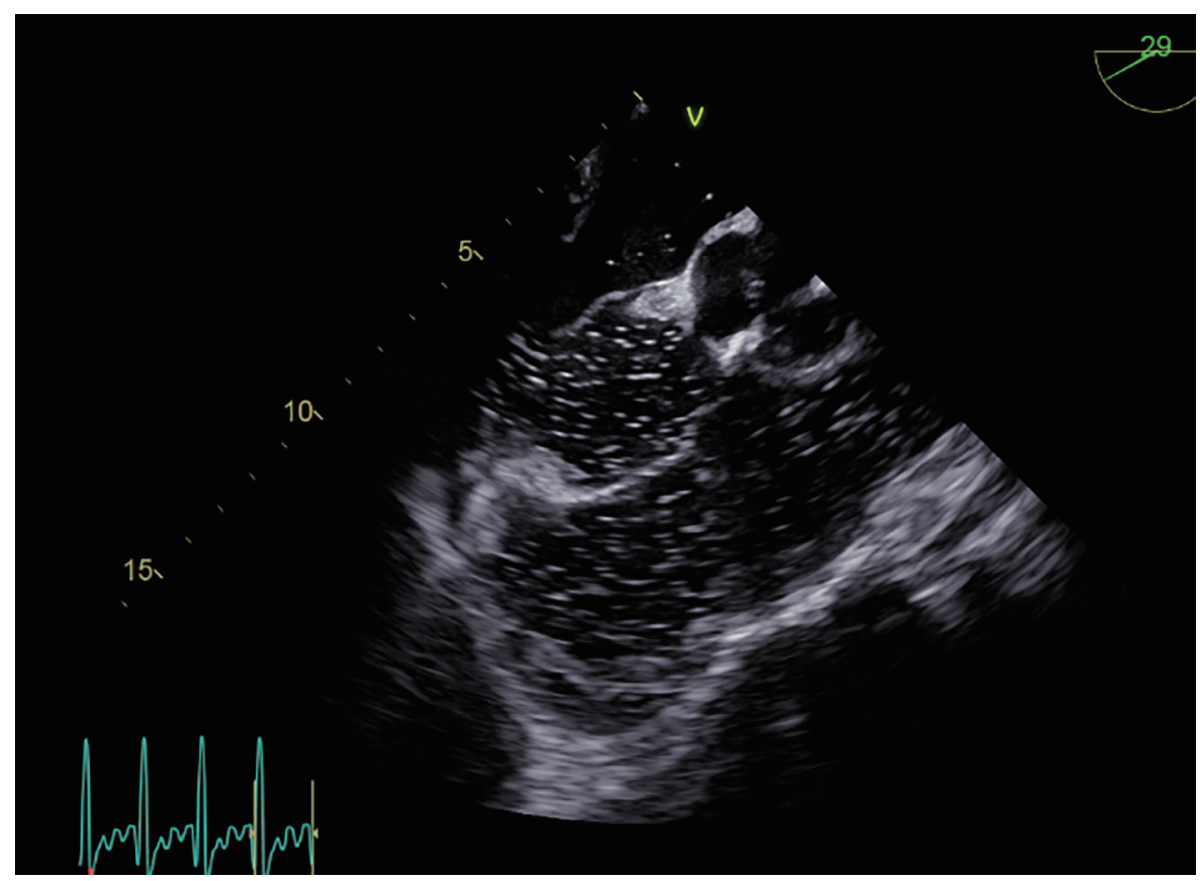

Figure 4. Transesophageal echocardiography at the level of the aortic valve with the scope rotated to $29^{\circ}$ during a bubble study, revealing an intra-atrial septal aneurysm with a small patent foramen ovale and a right-to-left shunt as evidenced by the bubbles within the left atrium. 
associated with other malformations of right heart structures, including pulmonary artery stenosis or atresia, tricuspid valve abnormalities, atrial septal defects, and/or Ebstein anomaly. Cyanosis, however, is a very rare presentation of this condition. Even though our patient had an accompanying patent foramen ovale, this was small in size and would not have resulted in significant right to left shunting or cyanosis.

Detection of CTD is often based on echocardiography. However, magnetic resonance imaging (MRI) was previously found to have a higher detection rate $(95 \%)$ when compared with echocardiography and cardiac angiography in the evaluation of pulmonary venous anomalies, which included cases of cor triatriatum (69\% for angiography and 38\% for echocardiography) [4-7]. Further studies are needed to evaluate detection rates between transthoracic and transesophageal echocardiography.

Right heart catheterization may reveal elevated pressure in the proximal right atrial chamber. It is also capable of detecting a gradient across the accessory membrane. One proposed complication with right heart catheterization is the entrapment of the guidewire or catheter within the fenestrations. Therefore, care must be paid with procedures within the right atrium of such patients. Other complications include misplacement, or leftward bowing of atrial devices for management of concomitant atrial septal defects. In addition, proper deployment and device stability might be prevented, overlap of the valve remnant, and underor oversizing of devices could occur. This is due to the CTD membrane obstructing the view and preventing adequate definition of the margins of an associated atrial septal defect $[8,9]$.

Asymptomatic patients are generally not treated unless they are undergoing cardiac surgery for other reasons. In symptomatic patients, control of fluid retention and ratecontrol of atrial arrhythmias (e.g. atrial fibrillation) may be required. Inotropic agents may be needed in patients with severe right sided heart failure. In the past, the mainstay of treatment for symptomatic patients was surgical resection of the dividing membrane [7]. Recently, however, percutaneous catheter disruption of the membrane has been suggested as a preferred alternative to open heart surgery $[10,11]$.

\section{Conclusion}

CTD is extremely rare; however, this diagnosis should be considered in patients presenting with symptoms of right-sided heart failure. Due to the association it has with right-sided congenital heart abnormalities, it should also be considered prior to interventions within the right atrium. CTD can be easily corrected percutaneously or by surgical excision of the membrane.

\section{Acknowledgments}

None to declare.

\section{Financial Disclosure}

None to declare.

\section{Conflict of Interest}

None to declare.

\section{Informed Consent}

Informed consent was obtained.

\section{Author Contributions}

GH: drafting article, writing manuscript; JS: writing manuscript, obtaining consent; SI: obtaining appropriate images and interpretation of images; PH: correspondence, general supervision, manuscript editing and manuscript review.

\section{Data Availability}

The authors declare that data supporting the findings of this study are available within the article.

\section{References}

1. Embrey RP. Cor triatriatum, pulmonary vein stenosis, atresia of the common pulmonary vein. In: Mavroudis C, Backer CL, editors. Pediatric cardiac surgery. 2nd ed. St Louis: Mosby-Year Book; 1994. pp. 503-504.

2. Arrants JE, Riopel DA, Catalano PW. Cor triatriatum: preoperative diagnosis and successful surgical correction in a ten-week-old infant. Chest. 1973;63(6):1027-1028.

3. Hoye DJ, Wilson EC, Fyfe DA, Guzzetta NA. Cor triatriatum dexter: a rare cause of neonatal cyanosis. Anesth Analg. 2010;110(3):716-718; discussion 718.

4. Burton DA, Chin A, Weinberg PM, Pigott JD. Identification of cor triatriatum dexter by two-dimensional echocardiography. Am J Cardiol. 1987;60(4):409-410.

5. Fiorilli R, Argento G, Tomasco B, Serino W. Cor triatriatum dexter diagnosed by transesophageal echocardiography. J Clin Ultrasound. 1995;23(8):502-504.

6. Masui T, Seelos KC, Kersting-Sommerhoff BA, Higgins CB. Abnormalities of the pulmonary veins: evaluation with MR imaging and comparison with cardiac angiography and echocardiography. Radiology. 1991;181(3):645649.

7. Ott DA, Cooley DA, Angelini P, Leachman RD. Successful surgical correction of symptomatic cor triatriatum dexter. J Thorac Cardiovasc Surg. 1979;78(4):573-575.

8. Martinez-Quintana E, Rodriguez-Gonzalez F. Focusing on cor triatriatum dexter and atrial septal defects. Tex Heart Inst J. 2014;41(5):567-568.

9. Vukovic PM, Kosevic D, Milicic M, Jovovic L, Stojanovic I, Micovic S. Cor triatriatum dexter and atrial septal defect in a 43-year-old woman. Tex Heart Inst J. 2014;41(4):418-420.

10. Savas V, Samyn J, Schreiber TL, Hauser A, O'Neill 
WW. Cor triatriatum dexter: recognition and percutaneous transluminal correction. Cathet Cardiovasc Diagn. 1991;23(3):183-186.
11. Haboub M, Drighil A. Successful balloon valvuloplasty of a subpulmonic membrane associated with cor triatriatum dexter: a case report. J Med Case Rep. 2019;13(1):291. 